\title{
Outcome of labor with epidural analgesia in primigravida at term
}

\author{
Reeti Mehra $^{1 *}$, Namrata Baweja ${ }^{2}$, Sukanya Mitra ${ }^{3}$ \\ ${ }^{1}$ Department of Obstetrics and Gynecology, Government Medical College and Hospital-32, Chandigarh, India \\ ${ }^{2}$ Department of Obstetrics and Gynecology, Baweja Multispeciality Hospital, Punjab, India \\ ${ }^{3}$ Department of Anaesthesia, Government Medical College and Hospital-32, Chandigarh, India
}

Received: 12 January 2019

Accepted: 06 February 2019

\section{*Correspondence:}

Dr. Reeti Mehra,

E-mail: mehrareeti1@gmail.com

Copyright: (C) the author(s), publisher and licensee Medip Academy. This is an open-access article distributed under the terms of the Creative Commons Attribution Non-Commercial License, which permits unrestricted non-commercial use, distribution, and reproduction in any medium, provided the original work is properly cited.

\begin{abstract}
Background: There is widespread acceptance of epidural analgesia among many physicians and patients, but disagreement remains regarding the effect of intrapartum epidural analgesia on the subsequent progress of labour and the mode of delivery. This study was designed to look into the effects of labour analgesia on maternal and fetal outcomes and compare the same with that of opioid analgesics.

Methods: It was a prospective randomized controlled trial on 100 patients where parturients were randomly allocated to either group 1-( $n=50$ patients) who received epidural analgesia or group $2(n=50)$ who received intramuscular tramadol. The two groups were compared for duration of labour, analgesic efficacy as assessed by using Visual Analogue Scale (VAS) ,type of delivery - normal/instrumental/operative interventions ,1 and 5 min neonatal Apgar score and neonatal breast-feeding behavior between the two groups.

Results: Epidural analgesic gave better pain relief than tramadol which was found to be stastically significant $(\mathrm{p}<0.001)$ on comparing the VAS scores and also gave statistically significantly higher satisfaction levels. Duration of both first and second stage of labor was comparable between the two groups and instrumental deliveries and caesarean sections were also comparable to that in tramadol group. There was a trend of higher neonatal Apgar scores in the epidural group than in the tramadol group.

Conclusions: Use of effective analgesia in labour, does not prolong second stage of labor and may facilitate good labour outcome both for the mother and the fetus and should be offered to patients in tertiary setups and equipped centres when the patients are apprehensive about labor pains.
\end{abstract}

Keywords: Epidural analgesia, Labour analgesia, Opiods, Tramadol

\section{INTRODUCTION}

The delivery of an alert and active baby into the arms of a conscious and pain free mother is one of the most exciting and rewarding experiences in medicine." is a famous quote by Moir. Ideal labour analgesia is considered to be one which provides adequate pain relief, has no side effects on either labour progression or mode of delivery, does not cause any neonatal complications and provides a pleasant and memorable experience to the mother. In the absence of a medical contraindication, maternal request is a sufficient medical indication for pain relief during labour. ${ }^{1}$

In many countries today, the availability of regional analgesia for labour is considered a reflection of standard obstetric care. The epidural acceptance is up to $60 \%$ in the major maternity centres of the US. ${ }^{2}$ In Canada, the epidural rate varies between the provinces from $30 \%$ to $69 \% .^{3}$ In our country, the awareness is still lacking. Over 
the past several years, due to extensive research and intensive medical and public health effort, the use of epidural analgesia has risen, however, disagreement remains regarding the effect of intrapartum epidural analgesia on the subsequent progress of labour and the mode of delivery. It is important that authors are aware of the risks and benefits of the technique, so that authors are better adept at guiding the patient through this stressful time. This study was designed specifically to look into the effects of labour analgesia on maternal and fetal outcomes and compare the same with that of opioid analgesics.

\section{METHODS}

The present study was conducted on 100 women by the Department of Obstetrics and Gynaecology, GMCH, Sector, 32 Chandigarh in collaboration with Department of Anaesthesiology of the same hospital. After the approval of study by Institutional ethics committee, a written informed consent was taken from the patient who satisfied the inclusion criteria.

\section{Inclusion criteria}

- Primigravida patients after 37 weeks of period of gestation with a singleton pregnancy ,cephalic presentation with spontaneous onset of labour.

\section{Exclusion criteria}

- Study were those with blood coagulation defects,

- infection at the anticipated site of puncture ,with hemodynamic instability,

- Twin gestation,

- Previously diagnosed uterine anomalies having any medical disorder like heart disease,

- Chronic renal disease, diabetes, auto immune diseases like SLE etc or women refusing for the procedure.

It was a prospective randomized controlled trial where parturients were randomly allocated to either group 1 or group 2, receiving epidural analgesia or intramuscular tramadol respectively Analgesia was administered at the first request for pain relief according to the group allotted after starting induction of labour.

In group 1, for insertion of the epidural catheter the patient was taken to the clean labour room (CLR) operation theatre. A baseline visual analogue score (VAS) was also obtained.

Epidural injection was given using midline technique with disposable 18G Tuohy's needle in the L2-3 or L3-4 interspace after giving local analgesic. The loading dose of $10 \mathrm{ml}$ of $0.1 \%$ bupivacaine with fentanyl $2 \mu \mathrm{g} / \mathrm{ml}$ was given as the test dose by an anaesthetist. Maintenance of PCEA (Master PCA pump, Fresenius Kabi Company) was started after the loading dose with either $10 \mathrm{ml}$ of
$0.1 \%$ bupivacaine with fentanyl $2 \mu \mathrm{g} / \mathrm{ml}$ as a continuous background infusion. The patients were provided with a remote controlled hand-held button and there was a provision of patient controlled boluses of $10 \mathrm{ml}$ of the same drug with a lockout interval of $15 \mathrm{mins}$ if needed (VAS>30). The patient was observed for 20mins and monitored continuously for HR, noninvasive $\mathrm{BP}, \mathrm{SpO} 2$, level of sensory block, pain score, time for the onset of action (VAS $<30)$ and modified Bromage score for motor power in the CLR OT before shifting the patient to CLR for monitoring of the mother and foetus during the course of labour.

In the event of an inadvertent intravascular or dural puncture by the epidural needle or catheter, the parturient was disqualified from recruitment into the study and managed according to the departmental protocols.

The following variables were monitored continuously after the block and recorded at following intervals:

- $\quad$ Systolic blood pressure (SBP) and HR every 5 min for the first $20 \mathrm{~min}$ and then every $1 \mathrm{~h}$ until delivery. Hypotension was defined as a decrease in SBP of more than $20 \%$ below baseline, and was treated with left uterine tilt, IV fluids, and vasopressors as necessary.

- Continuous fetal heart rate monitoring.

- VAS every $5 \mathrm{~min}$ for the first $20 \mathrm{~min}$ and then every $1 \mathrm{~h}$ until delivery.

- Sensory block at midline (loss of cold sensation to ice) every $5 \mathrm{~min}$ for the first $20 \mathrm{~min}$ and then every 1 $\mathrm{h}$ until delivery.

- Lower limb motor block every 5 min using the modified Bromage scale $(0=$ no block, $1=$ unable to flex either hip joint but able to move knee and ankle joints, 2 = unable to move hip and knee of either limb but able to move either ankle, $3=$ unable to move hip, knee or ankle joint of either lower limb) for the first $20 \mathrm{~min}$ and then every $1 \mathrm{~h}$ until delivery.

- Side effects: shivering, nausea, vomiting, fetal bradycardia were recorded. The presence of an abnormality of the fetal heart tracing were diagnosed and assessed by an attending obstetrician who was blinded to the patient's group assignment.

- Cervical dilation and use of oxytocin were recorded. Time of delivery, duration of second stage of labour, mode of delivery, Apgar scores at 1 and 5 minute were also recorded.

- Overall maternal satisfaction scores with labour analgesia (VAS based) were also assessed and documented.

In group 2, tramadol was given intramuscular injection at dose of $1 \mathrm{mg} / \mathrm{Kg}$ at first request for pain relief. If the next dose required within 4 hours, half of the initial dose was administered with maximum dose of $200 \mathrm{mg}$ in 4 hours, if required after 4 hours same dose as initial bolus was given with maximum of $400 \mathrm{mg}$ in 24 hours. VAS (Visual Analogue Scale) was categorised in various 
categories to assess the pain scale of the patients before and after the use of analgesia.

\section{Outcomes measured}

Comparison of duration of labour between two group, Analgesic efficacy as assessed by using VAS score, Type of delivery Normal/Instrumental/Operative Interventions, Comparison of 1- and 5-min Apgar score, Comparison of neonatal breast-feeding behaviour between the two groups and satisfaction levels

\section{Follow up}

The mothers were kept under observation for 24 hours post-partum to assess any complications i.e. sedation, retention of urine, post-partum haemorrhage etc. The neonates were followed up till discharge from hospital and breast-feeding behaviour was studied. They were also followed at 4 weeks post-partum by means of contact numbers/address.

\section{Statistical analysis}

The statistical analysis was carried out using statistical package for social sciences (SPSS Inc., Chicago, IL, and
Version 15.0 for Windows). All quantitative variables were estimated using measures of central location (Mean, Median) and measures of dispersion (Standard Deviation and Standard Error).

Normality of data was checked by measures of skewness and Kolmogrov-Smirnov test of normality. For normally distributed data, means were compared using student's $\mathrm{t}$ test and for skewed data or scores, Mann Whitney test was applied.

Qualitative or categorical variables were described as frequencies and proportions. Proportions were compared using Chi square or Fischer's exact test, whichever was applicable.

All statistical tests were two sided and performed at a significance level of $\mathrm{p}=0.05$.

\section{RESULTS}

The mean age of the women in groupland 2 was $25.02 \pm 3.14$ years and $24.12 \pm 4.03$ years respectively, which was comparable in both the groups. Both the groups were comparable in terms of height, weight, BMI and period of gestation.

Table 1: VAS score after analgesia in both the groups.

\begin{tabular}{|c|c|c|c|c|c|}
\hline \multirow{2}{*}{\multicolumn{3}{|c|}{ VAS Score }} & \multicolumn{2}{|l|}{ Group } & \multirow{2}{*}{ Total } \\
\hline & & & Epidural & Tramadol & \\
\hline & \multirow{2}{*}{ 0.1-3.9 (Mild) } & Count & 42 & 2 & 42 \\
\hline & & $\%$ within group & $84 \%$ & $4.0 \%$ & $43.3 \%$ \\
\hline & \multirow{2}{*}{ 4-6.9 (Moderate) } & Count & 8 & 35 & 42 \\
\hline & & $\%$ within group & $16 \%$ & $70.0 \%$ & $43.3 \%$ \\
\hline & \multirow{2}{*}{ 7-9.9 (Severe) } & Count & 0 & 13 & 13 \\
\hline & & $\%$ within group & $.0 \%$ & $26.0 \%$ & $13.4 \%$ \\
\hline \multirow{2}{*}{\multicolumn{2}{|c|}{ Total }} & Count & 50 & 50 & 97 \\
\hline & & $\%$ within group & $100.0 \%$ & $100.0 \%$ & $100.0 \%$ \\
\hline
\end{tabular}

Table 2: VAS score in second stage of labour.

\begin{tabular}{|c|c|c|c|c|c|}
\hline & & & \multicolumn{2}{|l|}{ Group } & \multirow{2}{*}{ Total } \\
\hline & & & Epidural & Tramadol & \\
\hline & \multirow{2}{*}{ 0.1-3.9 (Mild) } & Count & 32 & 0 & 32 \\
\hline & & $\%$ within group & $80.0 \%$ & $0 \%$ & $39.5 \%$ \\
\hline & \multirow{2}{*}{ 4-6.9 (Moderate) } & Count & 8 & 10 & 18 \\
\hline & & $\%$ within group & $20.0 \%$ & $24.3 \%$ & $22.2 \%$ \\
\hline & \multirow{2}{*}{ 7-9.9 (Severe) } & Count & 0 & 31 & 31 \\
\hline & & $\%$ within group & $0 \%$ & $75.6 \%$ & $38.2 \%$ \\
\hline \multirow{2}{*}{\multicolumn{2}{|c|}{ Total }} & Count & 40 & 41 & 81 \\
\hline & & $\%$ within group & $100.0 \%$ & $100.0 \%$ & $100.0 \%$ \\
\hline
\end{tabular}

The mean BMI was $23.58 \pm 2.19$ in group1 and in the tramadol group it was 22.64 $\pm 2.5 \quad(\mathrm{p}>0.05)$ The distribution of the patients in both the groups according to their habitat i.e. rural and urban was also comparable. 
The analgesia was given in patients complaining of severe pain (Grade IV VAS). Only 1 woman in Tramadol group received analgesia at moderate pain. Median VAS Scores before analgesia was 9 and 8 in the epidural and tramadol groups respectively and the difference was not statistically significant. It was found that epidural analgesia gave better pain relief compared to the tramadol group. More than $80 \%$ of the patients with Grade IV VAS Score before analgesia had a Grade II VAS Score after the use of epidural analgesia. This was statistically significant $(\mathrm{p}<0.05)$. Only $4 \%$ of the patients in Tramadol group complained of mild pain whereas $96 \%$ of the women still complained of moderate to severe pain. The pain score at maximum relief are shown in table 1 .

Table 2 represents the pain scores perceived by women in second stage of labour. 19 patients in the study underwent caesarean section before reaching the second stage of labour and were excluded from this analysis. Two women had caesarean section in second stage of labour and their pain score was included. About $80 \%$ women in the epidural group had VAS scores less than 4 as compared to none in Tramadol group. Median VAS Scores was 3 and 8 in epidural and tramadol groups respectively. This difference was found to be highly significant $(\mathrm{p}<0.01)$. The VAS Scores in the epidural group were significantly lower than the VAS scores in the tramadol group $(\mathrm{p}<0.01)$. The mean duration of active stage was $13.16 \pm 2.8$ hours in the epidural group and $12.75 \pm 2.9$ hours in the tramadol group. In both groups $70 \%$ women delivered within 16 hours.The duration of active stage of labour was comparable between the groups ( $p>0.05$ ). Mean duration of second stage of labour was $69.90 \pm 5.2$ mins in epidural group and $85 \pm 18.8$ mins in tramadol group (Table 3 ).

Table 3: Duration of second stage of labour in both the groups.

\begin{tabular}{|c|c|c|c|c|c|}
\hline & & & \multicolumn{2}{|l|}{ Group } & \multirow{2}{*}{ Total } \\
\hline & & & Epidural & Tramadol & \\
\hline \multirow{10}{*}{ Duration of labour } & \multirow{2}{*}{$\leq 30$} & Count & 15 & 3 & 18 \\
\hline & & $\%$ within group & $39 \%$ & $8 \%$ & $23 \%$ \\
\hline & \multirow{2}{*}{$31-60$} & Count & 13 & 11 & 26 \\
\hline & & $\%$ within group & $34 \%$ & $27 \%$ & $33 \%$ \\
\hline & \multirow{2}{*}{$61-120$} & Count & 6 & 25 & 32 \\
\hline & & $\%$ within group & $16 \%$ & $63 \%$ & $41 \%$ \\
\hline & \multirow{2}{*}{$121-180$} & Count & 2 & 2 & 4 \\
\hline & & $\%$ within group & $5 \%$ & $5 \%$ & $5 \%$ \\
\hline & \multirow{2}{*}{$>180$} & Count & 2 & 0 & 2 \\
\hline & & $\%$ within group & $5 \%$ & $0 \%$ & $3 \%$ \\
\hline \multirow{2}{*}{\multicolumn{2}{|c|}{ Total }} & Count & 38 & 41 & 79 \\
\hline & & $\%$ within group & $100.0 \%$ & $100.0 \%$ & $100.0 \%$ \\
\hline
\end{tabular}

Table 4: Indications for caesarean section in both the groups.

\begin{tabular}{|c|c|c|c|c|c|}
\hline & & & \multicolumn{2}{|l|}{ Group } & \multirow{2}{*}{ Total } \\
\hline & & & Epidural & Tramadol & \\
\hline & \multirow{2}{*}{ Deep transverse arrest } & Count & 0 & 1 & 1 \\
\hline & & $\%$ within group & $0 \%$ & $9.1 \%$ & $4.8 \%$ \\
\hline & \multirow{2}{*}{ Fetal bradycardia } & Count & 0 & 6 & 6 \\
\hline & & $\%$ within group & $0 \%$ & $18.2 \%$ & $9.5 \%$ \\
\hline & \multirow{2}{*}{ Second stage Arrest } & Count & 2 & 0 & 2 \\
\hline & & $\%$ within group & $20.0 \%$ & $27 \%$ & $10 \%$ \\
\hline & \multirow{2}{*}{ Meconium stained liquor } & Count & 3 & 3 & 6 \\
\hline & & $\%$ within group & $30 \%$ & $2 \%$ & $29 \%$ \\
\hline & \multirow{2}{*}{ Non-Progress of labour } & Count & 5 & 1 & 6 \\
\hline & & $\%$ within group & $50.0 \%$ & $9.1 \%$ & $28.6 \%$ \\
\hline \multirow{2}{*}{\multicolumn{2}{|c|}{ Total }} & Count & 10 & 11 & 21 \\
\hline & & $\%$ within group & $100.0 \%$ & $100.0 \%$ & $100.0 \%$ \\
\hline
\end{tabular}


$48 \%$ of women in Epidural group and $3.4 \%$ in Tramadol group delivered within 60 minutes. There were 4 women in epidural group and 2 women in Tramadol group who had second stage longer than 2 hours. This result was not statistically significant $(\mathrm{p}>0.05)$.

Two patients from the study had caesarean section in the second stage of labour and they were excluded from this analysis. In epidural group, $66 \%$ delivered normally compared to $68 \%$ in the tramadol group. $14 \%$ women in epidural group and $10 \%$ in the tramadol group had instrumental delivery.

This result was not statistically significant. Incidence of Caesarean sections was $20 \%$ in epidural group and $22 \%$ in tramadol group. This mode of delivery was comparable in both the groups.

Table 4 shows the indications of Caesarean section in both the groups and it was found out that the primary indication in the epidural group was Non-Progression Of Labour, which comprised $50 \%$ of the cases, whereas the primary indication in the tramadol group was fetal bradycardia, amounting to $54 \%$ of the total cases.

Table 5 shows the indications for instrumental delivery. The commonest was fetal distress in second stage in both the groups. There were $14 \%$ instrumental deliveries in the epidural group and $10 \%$ in tramadol group which were statistically comparable $(\mathrm{p}=0.287)$.

Table 5: Indications for instrumental delivery in both the groups.

\begin{tabular}{|c|c|c|c|c|c|}
\hline & & & \multicolumn{2}{|l|}{ Group } & \multirow[b]{2}{*}{ Total } \\
\hline & & & Epidural & Tramadol & \\
\hline \multirow{4}{*}{ Indication } & \multirow{2}{*}{ Fetal Distress } & Count & 5 & 3 & 8 \\
\hline & & $\%$ within group & $71 \%$ & $60 \%$ & $66 \%$ \\
\hline & \multirow{2}{*}{ Prolonged II Stage } & Count & 2 & 2 & 4 \\
\hline & & $\%$ within group & $29 \%$ & $40 \%$ & $34 \%$ \\
\hline \multirow{2}{*}{\multicolumn{2}{|c|}{ Total }} & Count & 7 & 5 & 12 \\
\hline & & $\%$ within group & $100.0 \%$ & $100.0 \%$ & $100.0 \%$ \\
\hline
\end{tabular}

Most of the neonates weighed between $2.5-3.5 \mathrm{~kg}$ with almost comparable distribution in both the groups. 9 neonates had Apgar score of less than 9in the tramadol group.

The trend of higher Apgar Score was found more in the epidural group and the difference was found to be statistically significant $(\mathrm{p}<0.05)$. Only one neonate in epidural group had Apgar $<9.1$ neonate in the epidural group and 9 in the tramadol group needed bag and mask ventilation. This result was found to be statistically significant. None of the neonates needed Naloxone or had sepsis. Satisfaction levels were also analysed. Excellent to very good analgesia was reported in $88 \%$ of the cases in the epidural group as compared to $24 \%$ in the tramadol group.

This result was statistically significant ( $\mathrm{p}<0.01)$. Following 12-24 hours of delivery, women were also questioned regarding the future desire for using the same analgesia in the next pregnancy. More than $70 \%$ patients who received epidural analgesia expressed a desire to use it again in the next pregnancy as compared to only $30 \%$ in the tramadol group. This was also statistically significant $(\mathrm{p}<0.01)$.

The time of initiation of breast feeding was recorded from the time of delivery. In the epidural group the mean time of initiation of breast feeding was $1.9 \pm 0.2$ hours compared with $1.5 \pm 0.5$ hours in the tramadol group which was comparable. In the epidural group $79.2 \%$ neonates and $53 \%$ neonates in the Tramadol group were reported to have established breast feeding within 24 hours of delivery whereas $20.8 \%$ in epidural and $47 \%$ in tramadol took more than 24 hours. The breast-feeding pattern at 4 weeks post-partum was comparable between the two groups and the difference was not statistically significant $(\mathrm{p}=0.484)$. 5 women from the epidural group and 3 from the tramadol group were lost to follow up.

There were no maternal complications. In epidural group $7(14 \%)$ women had short lasting reversible numbness in limbs but none had motor weakness. The most common complication in the tramadol group was vomiting reported in $23(46 \%)$ women but none in the epidural group.

\section{DISCUSSION}

There is a general agreement that a patient in labor should be given the option to have an epidural block for pain management. Despite this consensus, there are differences in practice patterns as to when to initiate an epidural and how to minimize its impact on the duration and outcome of a patient's labor. A review of the literature suggests conflicting results on whether epidural analgesia prolongs stages of labor, increases instrumental and cesarean delivery rates which is the 
most often discussed controversy associated with epidural analgesia. It is evident from our study that epidural analgesia is much more superior to tramadol for analgesia in both, first as well as second stages of labor (tables I,II). With Tramadol, majority of women had onset of analgesic effect in 15 to 30 mins whereas maximum analgesic effect for all women in epidural group was achieved in 6-10 minutes . Shorter onset of action by epidural analgesia was clinically significant as it relieved maternal anxiety earlier and developed faith in the efficacy of therapy. Authors initiated epidural on the woman's first request for pain which was when the VAS score was. ${ }^{8-9}$ A Cochrane review on 15,752 women showed no clinically meaningful difference in duration of labor, instrumental birth, caesarean section with early versus late initiation of epidural analgesia. ${ }^{4}$

The effect of epidural analgesia on the progress of labor has been extensively studied and has conflicting results. Anim-Souman and colleagues performed a Cochrane review of epidural analgesia effects in labor using 38 trials involving 9658 parturients. ${ }^{5}$ Although there were no significant differences in the length of the first stage of labor, second stage was lengthened by an average 15 minutes. The clinical significance of such a limited prolongation is debatable.

In the same Cochrane review, 23 randomized trials $(\mathrm{N}=7935)$ were analyzed comparing operative (forceps or vacuum-assist) deliveries in relation to epidural analgesia. Operative vaginal delivery was linked to epidural analgesia (RR 1.42; 95\%CI 1.28-1.57).5 Several theories of possible etiologies include local anesthetic agents and narcotics interference with normal expulsive efforts via suppression of the bear-down reflex and failure of appropriate time to allow internal rotation of the fetal head. This is in contrast to the study by Wang et al. ${ }^{6}$ More recently, a systematic review of 6 studies $(\mathrm{N}=15,399)$ showed no increased risk of cesarean (pooled risk ratio $1.0295 \%$ CI 0.96-1.08) or instrumental (pooled risk ratio $0.9695 \%$ CI $0.89-1.05$ ) delivery for women receiving early epidural (defined as $3 \mathrm{~cm}$ or less) in comparison with late epidural placement. ${ }^{7}$

Wassen et al8 suggested that routine epidural analgesia may increase the rate of operative deliveries, however the difference between vaginal (difference: $4.5 \%$ (95\%CI: $1.6,10.6))$ or via cesarean $(3.6 \%(95 \% \mathrm{CI}:-3.1,10.3) \mathrm{did}$ not reach statistical significance. Also, adverse labor outcomes such as incidence of shoulder dystocia, postpartum hemorrhage, manual placenta extraction, and third/fourth degree perineal lacerations; and neonatal outcomes were no different. However, maternal hypotension and motor blockade was significant in the routine epidural analgesia group . In present study, in the epidural group, 7 (14\%) women had numbness in limbs but none had motor weakness. The most common complication in the tramadol group was vomiting reported in $23(46 \%)$ women but none in the epidural group. There was no incidence of respiratory depression or sedation in any women of either group.

These heterogeneous results on labor could be because of difference in labour management and analgesic administration protocol. Present study was carried out in women having spontaneous labour where oxytocin was given for augmentation of labour and epidural analgesia was withdrawn in second stage and tramadol was stopped after $8 \mathrm{~cm}$ dilation, to facilitate bearing down efforts . Analgesia was initiated on maternal demand when VAS score was 8-9. These factors may be responsible for comparable duration of first and second stage of labour in both groups. In present study, instrumental deliveries and caesarean sections were also comparable to that in tramadol group explicably for the same reason. In present study, epidural analgesia resulted in far better analgesia and majority of women in that group rated its effect as excellent. They were interested in using the same in future pregnancy also. However, only a small number in tramadol rated it to be excellent, and the rest were dissatisfied with its effects.

The most critical factors for breast feeding success are analgesia, support, good labour experience and educating the mother. Epidural analgesia by providing better analgesia probably reduced maternal exhaustion allowing better maternal participation in establishing breast feeding and bonding. Authors found no significant difference in breastfeeding patterns at initiation or 4 weeks postpartum follow up similar to Gizzo and French CA et al. ${ }^{9,10}$ There are very few Indian studies on epidural analgesia as it is relatively less frequently used in our country. Recently Aggarwal et al showed that epidural analgesia by ropivacaine in Indian nulliparous resulted in shorter duration of first stage and prolonged duration of second stage of labour compared with parturients without analgesia without any increase instrumental vaginal or caesarean delivery rate. ${ }^{11}$ This was similar to the study by Fyneface-Ogan et al who also showed similar results and postulated that short duration of first stage may be because of better analgesia with epidural resulting to decrease inhibitory effect of catecholamines on uterine contractility hence faster cervical dilatation. ${ }^{12}$ Regarding effect on neonates, Shrestha et al from India also demonstrated that epidural analgesia does not have any effect on the newborns in regard to breast feeding and birth asphyxia but did have effects like delayed passage of urine and increased incidence of instrumentation. ${ }^{13}$

\section{CONCLUSION}

To conclude, it is justified to suggest that facilities for epidural analgesia may not be available everywhere in developing countries like India and there are very few studies from India, but it is a very effective analgesia exhibiting high level of satisfaction and may facilitate good labour outcome both for the mother and the fetus. 
If epidural analgesia is withdrawn in second stage, effective analgesia does persist and has the advantage that, the second stage does not get prolonged . Epidural analgesia is safe and should be suggested in labour where the patients are apprehensive about the pain they might have to undergo.

\section{Funding: No funding sources}

Conflict of interest: None declared

Ethical approval: The study was approved by the Institutional Ethics Committee

\section{REFERENCES}

1. Caton D. The history of obstetric anesthesia. In: Chestnut DH, Polley LS, Tsen LC, Wong CA, eds. Obstetric Anesthesia Principles and Practice. 4th ed. Philadelphia: Elsevier Mosby; 2009:3-13.

2. Osterman MJ, Martin JA. Epidural and spinal anesthesia use during labor: 27-state reporting area, 2008. Natl Vital Stat Rep. 2011;59(5):1-13.

3. Canadian Institute of Health Information. Highlights of 2008-2009: Selected indicators describing the birthing process in Canada. Available from: http://www.cihi.ca161:670-5.

4. Sng BL, Leong WL, Zeng Y, Siddiqui FJ, Assam PN, Lim Y, Chan ES, Sia AT. Early versus late initiation of epidural analgesia for labour. Cochrane Database Syst Rev. 2014;(10):CD007238.

5. Anim-SM, Smyth RM, Jones L. Epidural versus nonepidural or no analgesia in labour ;Cochrane Database Syst Rev. 2011;(12)

6. Wang F, Shen X, Guo X, Peng Y, Gu X. Epidural analgesia in the latent phase of labor and the risk of cesarean delivery. Anesthesiology. 2009;111(4):87180

7. Wassen MM, Zuijlen J, Roumen FJ, Smits LJ, Marcus MA, Nijhuis JG. Early versus late epidural analgesia and risk of instrumental delivery in nulliparous women: A systematic review. BJOG. 2011;118(6):655-61.

8. Wassen MM, Smits LJ, Scheepers HC, Marcus MA, Van NJ, Nijhuis JG, Roumen FJ . Routine labour epidural analgesia versus labour analgesia on request: a randomized non-inferiority trial. BJOG. 2015;122(3):344-50.

9. Gizzo S, Di Gangi S, Saccardi C, Patrelli TS, Paccagnella G, Sansone L et al. Epidural analgesia during labor: impact on delivery outcome, neonatal well-being, and early breastfeeding; Breastfeed Med. 2012;7(4):262-8.

10. French CA, Cong X, Chung KS. Labor Epidural Analgesia and Breastfeeding: A Systematic Review. J Hum Lact. 2016;32(3):507-20.

11. Agrawal D, Makhija B, Arora M, Haritwal A, Gurha P. The effect of epidural analgesia on labour, mode of delivery and neonatal outcome in nullipara of India, 2011-2014 J Clin Diagn Res. 2014;8(10):OC03

12. S Fyneface-Ogan, CN Mato, SE Anya. Epidural anesthesia: views and outcomes of women in labour in a Nigerian hospital. Ann Afr Med. 2009;8(4):2506.

13. Shrestha B, Devgan A, Sharma M . Effects of maternal epidural analgesia on the neonate-a prospective cohort study. Ital J Pediatr. 2014;40(1):99.

Cite this article as: Mehra R, Baweja N, Mitra S. Outcome of labor with epidural analgesia in primigravida at term. Int J Reprod Contracept Obstet Gynecol 2019;8:1055-61. 